\title{
High data density and capacity in chipless radiofrequency identification (chipless-RFID) tags based on double-chains of S-shaped split ring resonators (S-SRRs)
}

\author{
Cristian Herrojo, Javier Mata-Contreras, Ferran Paredes, and Ferran Martín* \\ CIMITEC, Departament d'Enginyeria Electrònica, Universitat Autònoma de Barcelona, 08193 Bellaterra, Spain
}

Received: 24 July 2017 / Received in final form: 7 October 2017 / Accepted: 10 October 2017

\begin{abstract}
The data density per surface (DPS) is a figure of merit in chipless radiofrequency identification (chipless-RFID) tags. In this paper, it is demonstrated that chipless-RFID tags with high DPS can be implemented by using double-chains of S-shaped split ring resonators (S-SRRs). Tag reading is achieved by near-field coupling between the tag and the reader, a CPW transmission line fed by a harmonic signal tuned to the resonance frequency of the S-SRRs. By transversally displacing the tag over the CPW, the transmission coefficient of the line is modulated by tag motion. This effectively modulates the amplitude of the injected (carrier) signal at the output port of the line, and the identification (ID) code, determined by the presence or absence of S-SRRs at predefined and equidistant positions in the chains, is contained in the envelope function. The DPS is determined by S-SRR dimensions and by the distance between S-SRRs in the chains. However, by using two chains of S-SRRs, the number of bits per unit length that can be accommodated is very high. This chipless-RFID system is of special interest in applications where the reading distance can be sacrificed in favor of data capacity (e.g., security and authentication). Encoding of corporate documents, ballots, exams, etc., by directly printing the proposed tags on the item product to prevent counterfeiting is envisaged.
\end{abstract}

Keywords: chipless RFID / coplanar waveguide / split ring resonators / high data capacity / security applications

\section{Introduction}

Transmission lines loaded with split ring resonators (SRRs) and with other electrically small resonators [1-5] have been used in numerous microwave applications, including filters [6,7], enhanced bandwidth components [8], multiband components [9-12], microwave sensors [13-30] and chipless radiofrequency identification (chipless-RFID) tags [31,32], among others. Concerning chipless RFID tags, the interest in this work, transmission lines loaded with multiple resonant elements, each tuned to a different frequency, have been proposed as multi-resonant frequency domain based tags [31-44]. In such chipless-RFID systems, the interrogation signal is a multi-frequency sweeping signal covering the spectral bandwidth of the resonant elements, and the ID code is inferred from the dips present in the frequency response (retransmission based tags) [3137 or in the radar cross section response (backscattered tags) [38-44], caused by the resonant elements. Therefore, the presence or absence of dips at predefined frequencies (each one corresponding to a different bit) is associated

\footnotetext{
* e-mail: Ferran.Martin@uab.cat
}

with the logic state ' 1 ' or ' 0 '. In general, the data capacity of these frequency-domain based tags is limited due to the required spectral bandwidth necessary to accommodate a large number of bits. Multi-state multi-resonator tags, where more than one bit of information is associated to each resonant element, have been proposed to increase the density of bits per frequency and per surface [36,37]. Another strategy consists of using more than one domain (hybrid tags) [45-49], e.g., encoders based on frequency position and polarization diversity [47], or encoders where frequency domain is combined with phase deviation [46].

Despite the efforts to increase the number of bits with the previous frequency-domain and hybrid chipless-RFID systems, the reported tags do not exhibit the data capacity of chiped tags. Another approach, based on time domain, was recently reported by the authors [50,51]. Within this approach, tags consist of a chain of identical resonant elements etched or printed on a dielectric substrate. The presence or absence of resonant elements at predefined and equidistant positions determines the logic state ' 1 ' or ' 0 ', and the number of bits is only limited by the area occupied by the tag, since the spectral bandwidth is virtually null in these tags. Therefore, high data capacity can be achieved 


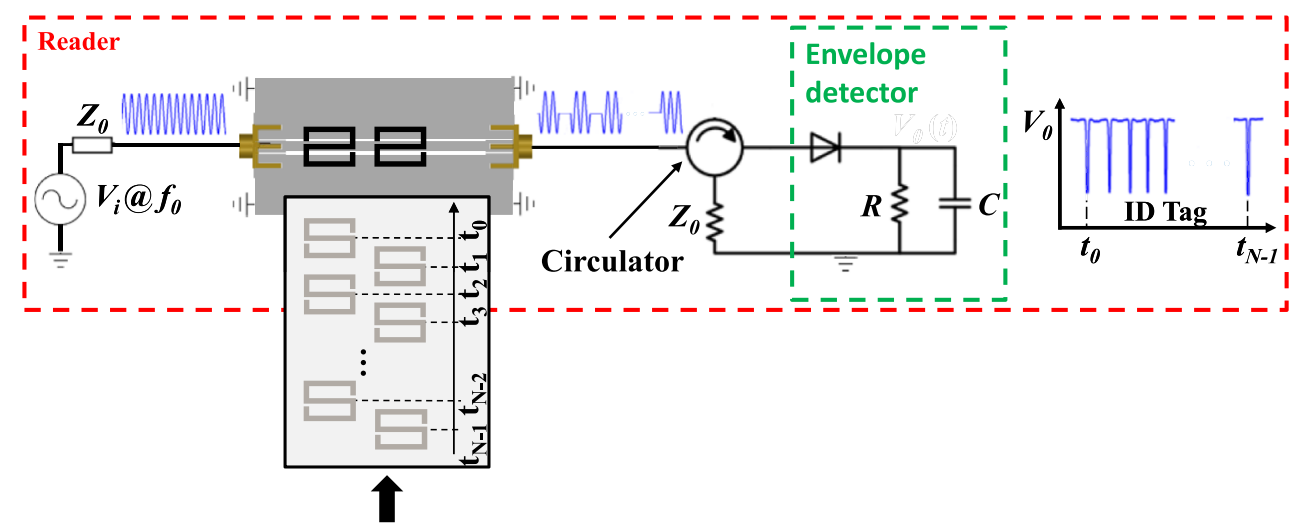

Fig. 1. Illustration showing the working principle of the proposed chipless-RFID system. A double chain of S-SRRs can be appreciated in the tag, according to the strategy to increase the data capacity per unit length proposed in the paper.

a)

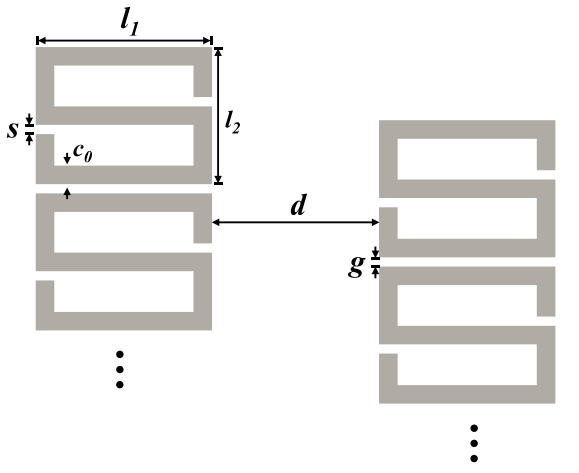

b)

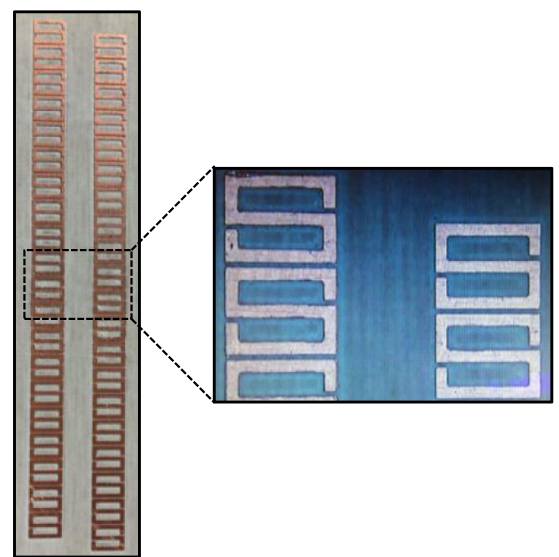

Fig. 2. Layout (zoom) of the double chain of S-SRRs (a) and photograph of the fabricated 40-bit chipless RFID tag (b). S-SRR dimensions are (in mm) $l_{1}=3.8, l_{2}=2.96, c_{0}=0.4, s=0.2, g=0.2$ and $d=3.6$.

with this novel time-domain chipless-RFID systems. Nevertheless, in order to reduce the space occupied by the tags as much as possible, it is important to optimize the data density per surface (DPS), accommodating the largest possible number of resonant elements in a certain area.

In [50,51], the resonant elements are S-shaped split ring resonators S-SRRs. Tag reading proceeds sequentially through tag motion, by transversally displacing the tag over a coplanar waveguide transmission line fed by a harmonic signal tuned to the resonance frequency of the SSRRs. By this means, the transmission coefficient of the line is modulated through near-field coupling between the line and the resonant elements. That is, each time an S-SRRs is aligned with the line axis, line-to-resonator coupling is maximized and the transmission coefficient is consequently minimized. This variable coupling, dictated by the presence or absence of resonant elements in the chain as the tag is displaced, effectively modulates the amplitude of the feeding (carrier) signal at the output port of the line, and the identification (ID) code is contained in the envelope function of the amplitude modulated (AM) signal. By using a simple envelope detector, the tag ID can thus be inferred. Figure 1 illustrates the working principle of this time-domain chipless RFID system based on near-field coupling.
In [51], 40-bit tags, occupying an area of $5.34 \mathrm{~cm}^{2}$ and a chain length of $14 \mathrm{~cm}$, were demonstrated. In this paper, we have doubled the number of bits, without significantly increasing the tag length (the critical dimension) by using a double-chain of resonant elements. The strategy to implement the double chain and the necessary modifications of the CPW transmission line (reader) are reported in this paper. The proposed system is validated by reading several fabricated tags with our experimental setup, and the ID code is obtained by visualizing the envelope function in an oscilloscope.

\section{Tag and reader design and fabrication}

Concerning the tags, they are based on the resonant elements used in [50,51], i.e., S-SRRs [52-54]. However, a double chain of S-SRRs is considered in this paper, with a relative displacement between S-SRR chains of half a chain period. The dimensions of the S-SRRs are those considered in $[50,51]$. A detail of the layout of the double S-SRR chain is depicted in Figure 2(a), whereas Figure 2(b) shows the picture of the 40-bit fabricated encoder with all the resonant elements present. The considered substrate for 


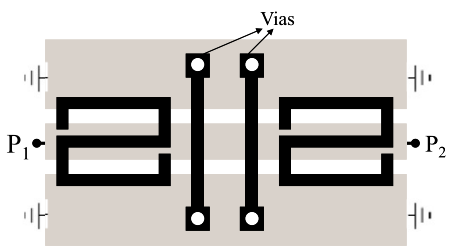

a)

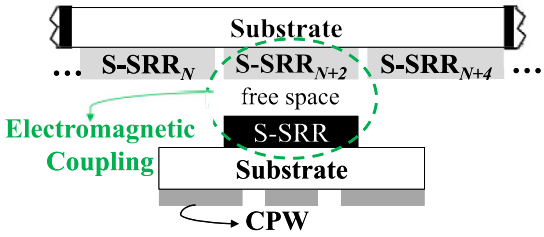

b)

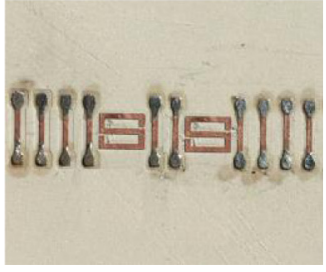

c)

Fig. 3. Layout (a), cross sectional view including the tag (b) and photograph (c) of the designed and fabricated S-SRR-loaded CPW acting as active part of the reader. In (c) only the backside of the CPW is shown.

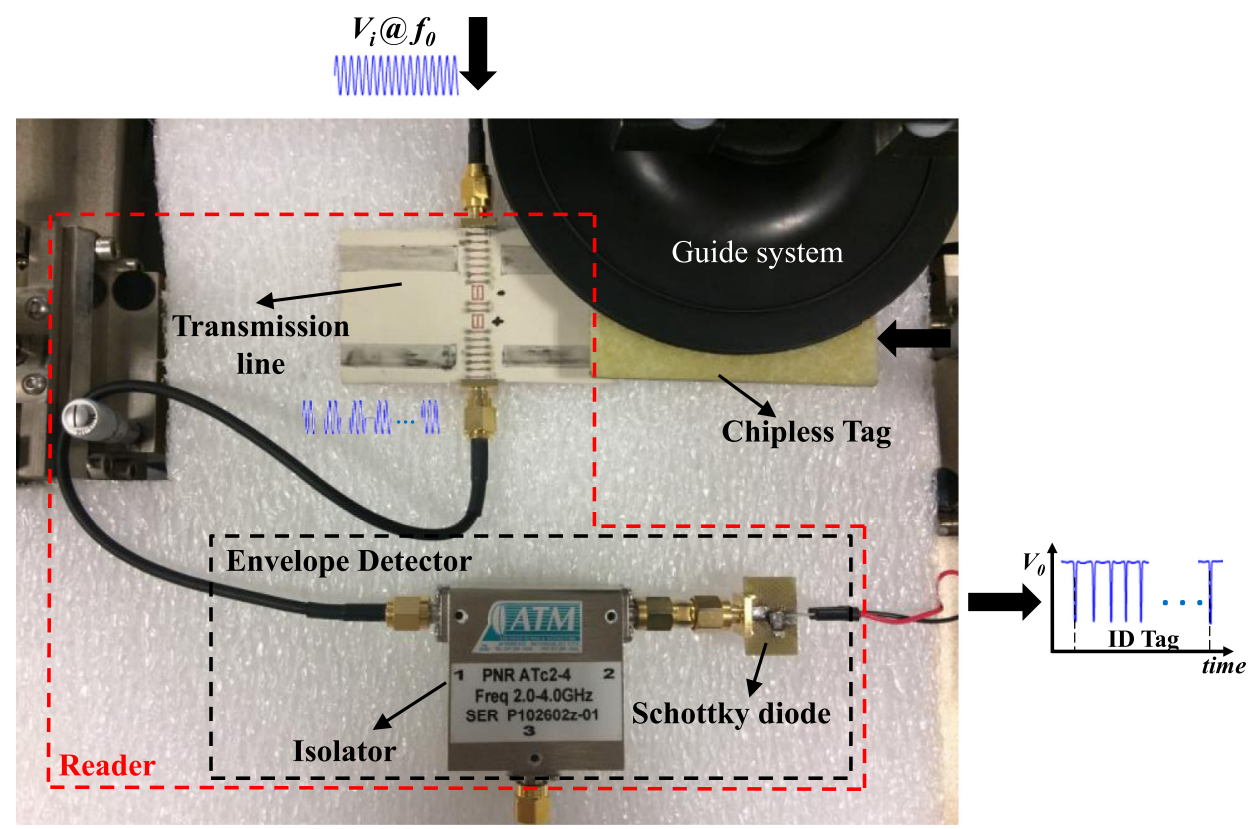

Fig. 4. Photograph of the experimental set-up.

tag implementation is the Rogers $R 04003 C$ with thickness $h=203 \mu \mathrm{m}$, dielectric constant $\varepsilon_{r}=3.55$ and tan $\delta=0.0021$.

Let us now consider the design of the CPW transmission line of the reader. In [50,51], a S-SRR identical to those of the tag was etched in the back substrate side of the $\mathrm{CPW}$, a $50 \Omega$ line. The purpose of this resonant element etched in the reader side is to prevent from the appearance of inter-resonator coupling in the tag chain (through magnetoinductive waves [55-60]) as well as multiple couplings between the line and the S-SRRs of the chain [30]. The key aspect is that when one of the S-SRRs of the tag chain is perfectly aligned with the S-SRR of the reader, both particles can be viewed as a single particle, the broadside coupled S-SRR (BC-S-SRR), with smaller fundamental resonance. Thus, by tuning the frequency of the carrier feeding signal to the frequency of the BC-SSRR, or slightly higher, the above-cited extra couplings are avoided. The reason is that the resonance frequency of the individual S-SRR is substantially higher.

If two chains of S-SRRs are considered in the tag (with a relative displacement of half a unit cell), this means that, consequently, two S-SRRs must be etched in the backside of the CPW transmission line. The distance between the S-
SRRs in the CPW must be identical to the transverse distance between the S-SRR chains. Through this approach, if a S-SRR is etched in a certain predefined position, either in the outer or inner tag chain, the corresponding ' 1 ' state will be detected, since the coupling with the line is ensured. Figure 3 shows the layout, cross sectional view and photograph of the proposed and fabricated S-SRR-loaded CPW. The structure has been fabricated in the Rogers RO3010 with thickness $h=0.635 \mathrm{~mm}$, dielectric constant $\varepsilon_{r}=10.2$.

\section{Experimental set-up and validation}

The photograph of the complete system is depicted in Figure 4. The frequency of the carrier signal is set to $f_{c}=4 \mathrm{GHz}$, which is the one corresponding to the BC-SSRR by considering an air gap of $0.25 \mathrm{~mm}$. By varying the air gap distance, the resonance frequency of the BC-S-SRR, $f_{0}$, obviously changes. If the carrier frequency is chosen as $f_{c}=f_{0}$ for a certain gap distance, and the air gap increases significantly, it may give rise to reading errors. The reason is that $f_{0}$ increases, and hence the modulation index decreases. Nevertheless, some tolerance exists since the 


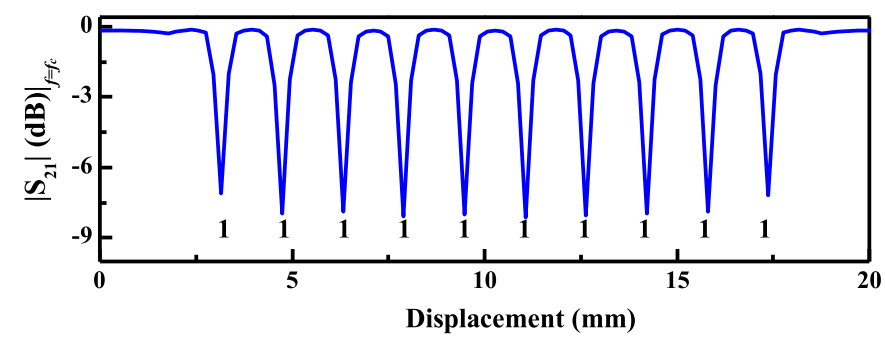

Fig. 5. Transmission coefficient at $f_{c}$, as the linear 10-bit tag is displaced above the S-SRR-loaded CPW transmission line, inferred from electromagnetic simulation using Keysight Momentum, by considering an aig gap of $0.25 \mathrm{~mm}$.

notch, centered at $f_{0}$, exhibits certain bandwidth. Moreover, we can fine tune the carrier frequency in order to match it to the resonance frequency of the BC-SRR.

Before the experimental validation (to be discussed next), we have obtained the transmission coefficient at $f_{c}$ through electromagnetic simulation, by considering a linearly shaped double S-SRR chain with all the SRRs present at the predefined positions (specifically, we have considered five S-SRRs in either chain, corresponding to a 10-bit encoder with ID code ' 1111111111 '). The simulated response, obtained by displacing the tag over the S-SRR loaded CPW and depicted in Figure 5, reveals that the tag functionality is achieved.

In practice, the carrier signal is generated by means of the Agilent E44338C function generator. The output port of the S-SRR-loaded CPW is connected to an isolator (implemented with the ATM ATc4-8 circulator) in order to prevent from mismatching reflections caused by the diode, an Avago HSMS-2860 device. Such diode is the essential part of the envelope detector, used for rectification purposes. Filtering is achieved by means of the N2795A active probe (with resistance $R=1 \mathrm{M} \Omega$ and capacitance $C=1 \mathrm{pF})$, connected to the Agilent MSO-X-3104A oscilloscope in order to visualize the envelope function providing the ID code.

Reading of three different encoders has been carried out by means of the proposed set-up. The envelope function of the 40-bit encoder with all resonators present at the predefined positions (code 1 of Fig. 6) has been modified to achieve codes 2 and 3 of Figure 6 . Specifically, we have cut some resonators, making them inoperative (i.e., equivalent to their absence, or ' 0 ' logic state) and hence programming the ID code corresponding to codes 2 and 3 of Figure 6. A drilling machine has been used to cut the required resonant elements along their symmetry plane. The data density per surface of the proposed tags is DPS $=5.53 \mathrm{bit} / \mathrm{cm}^{2}$, and the number of bits per unit length is $5.7 \mathrm{bit} / \mathrm{cm}$. Since tag reading proceeds sequentially, necessarily the longitudinal dimension of the proposed tags is much larger than the transverse dimension, the later being independent on the number of bits and given by the transverse size of the double SSRR chain. Therefore, the critical aspect, provided a large number of bits is required, is the number of bits per unit length. With the resulting density of bits per unit length,

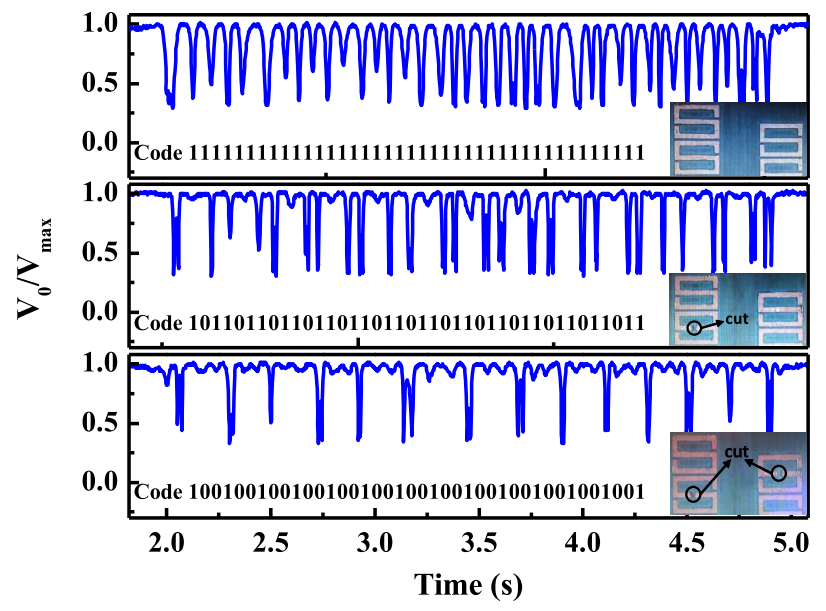

Fig. 6. Envelope functions of the 40-bit tags with the indicated codes.

it is possible to encode standard European documents (of size $29.7 \mathrm{~cm} \times 21.0 \mathrm{~cm}$ ) with 170 bits printed along the longer paper side.

\section{Conclusions}

In conclusion, a chipless-RFID system based on near-field and sequential bit reading, with tags implemented by double chains of S-SRRs, has been proposed. As compared to previous S-SRR based tags implemented with single chains of SRRs, the reported tags exhibit roughly twice the data density per unit length (i.e., $5.7 \mathrm{bit} / \mathrm{cm}$ ). The strategy to accommodate such high bit density in the tags, as well as the modifications in the reader (S-SRR-loaded CPW), necessary to properly read the tags, has been discussed.

The possibility to program the tags by detuning the resonant elements has been also demonstrated, and a proofof-concept, where the ' 0 ' state has been achieved by cutting the corresponding resonant elements, has been carried out. The reported high data capacity chipless-RFID system is contactless but needs proximity between the tag and the reader. It is thus of special interest in applications where a large number of bits is necessary, and read distance can be sacrificed. Encoding of secure documents, e.g., to avoid counterfeiting, is one of the envisaged applications. Work is in progress to implement the proposed tags in plastic and paper substrates.

This work was supported by MINECO-Spain (projects TEC201340600-R and TEC2016-75650-R), by Generalitat de Catalunya (project 2014SGR-157), by Institució Catalana de Recerca i Estudis Avançats (who awarded Ferran Martín), and by FEDER funds.

\section{References}

1. F. Martin, Artificial Transmission Lines for RF and Microwave Applications (John Wiley, Hoboken, NJ, USA, 2015)

2. R. Marques, F. Martín, M. Sorolla, Metamaterials with Negative Parameters: Theory, Design and Microwave Applications (John Wiley, Hoboken, NJ, USA, 2007) 
3. F. Martín, F. Falcone, J. Bonache, R. Marqués, M. Sorolla, Split ring resonator based left handed coplanar waveguide, Appl. Phys. Lett. 83, 4652 (2003)

4. F. Falcone, T. Lopetegi, J.D. Baena, R. Marqués, F. Martín, M. Sorolla, Effective negative- $\varepsilon$ stop-band microstrip lines based on complementary split ring resonators, IEEE Microw, Wireless Compon. Lett. 14, 280 (2004)

5. J.D. Baena, J. Bonache, F. Martín, R. Marqués, F. Falcone, T. Lopetegi, M.A.G. Laso, J. García, I. Gil, M. FloresPortillo, M. Sorolla, Equivalent circuit models for split ring resonators and complementary split rings resonators coupled to planar transmission lines, IEEE Trans. Microw. Theory Techn. 53, 1451 (2005)

6. J. Bonache, F. Martín, I. Gil, J. García-García, R. Marqués, M. Sorolla, Microstrip bandpass filters with wide bandwidth and compact dimensions, Microw. Opt. Technol. Lett. 46, 343 (2005)

7. J. Bonache, I. Gil, J. García-García, F. Martín, Novel microstrip band pass filters based on complementary split rings resonators, IEEE Trans. Microw. Theory Techn. 54, 265 (2006)

8. G. Sisó, J. Bonache, M. Gil, F. Martín, Application of resonant-type metamaterial transmission lines to the design of enhanced bandwidth components with compact dimensions, Microw. Opt. Technol. Lett. 50, 127 (2008)

9. J. Bonache, G. Sisó, M. Gil, A. Iniesta, J. García-Rincón, F. Martín, Application of composite right/left handed (CRLH) transmission lines based on complementary split ring resonators (CSRRs) to the design of dual band microwave components, IEEE Microw. Wireless Compon. Lett. 18, 524 (2008)

10. M. Durán-Sindreu, A. Vélez, F. Aznar, G. Sisó, J. Bonache, F. Martín, Application of open split ring resonators and open complementary split ring resonators to the synthesis of artificial transmission lines and microwave passive components, IEEE Trans. Microw. Theory Techn. 57, 3395 (2009)

11. F.J. Herraiz-Martínez, G. Zamora, F. Paredes, F. Martín, J. Bonache, Multiband printed monopole antennas loaded with open complementary split ring resonators for PANs and WLANs, IEEE Ant. Wirel. Propag. Lett. 10, 1528 (2011)

12. F.J. Herraiz-Martínez, F. Paredes, G. Zamora, F. Martín, J. Bonache, Dual-band printed dipole antenna loaded with open complementary split-ring resonators (OCSRRs) for wireless applications, Microw. Opt. Technol. Lett. 54, 1014 (2012)

13. J. Naqui, M. Durán-Sindreu, F. Martín, Novel sensors based on the symmetry properties of split ring resonators (SRRs), Sensors 11, 7545 (2011)

14. C. Mandel, B. Kubina, M. Schüßler, R. Jakoby, Passive chipless wireless sensor for two-dimensional displacement measurement, in: Proceedings of 41st European Microwave Conference, Manchester, UK, 2011, pp. 79-82

15. M. Puentes, C. Weiss, M. Schüßler, R. Jakoby, Sensor array based on split ring resonators for analysis of organic tissues, IEEE MTT-S Int. Microwave Symp. Dig., Baltimore, Maryland, June 2011

16. M.S. Boybay, O.M. Ramahi, Material characterization using complementary split-ring resonators, IEEE Trans. Instrum. Meas. 61, 3039 (2012)

17. C.-S. Lee, C.-L. Yang, Complementary split-ring resonators for measuring dielectric constants and loss tangents, IEEE Microw. Wireless Compon. Lett. 24, 563 (2014)
18. A. Ebrahimi, W. Withayachumnankul, S. Al-Sarawi, D. Abbott, High-sensitivity metamaterial-inspired sensor for microfluidic dielectric characterization, IEEE Sens. J. 14, 1345 (2014)

19. C.-L. Yang, C.-S. Lee, K.-W. Chen, K.-Z. Chen, Noncontact measurement of complex permittivity and thickness by using planar resonators, IEEE Trans. Microw. Theor. Technol. 64, 247 (2016)

20. L. Su, J. Mata-Contreras, P. Vélez, F. Martín, Estimation of conductive losses in complementary split ring resonator (CSRR) loading an embedded microstrip line and applications, in: IEEE MTT-S Int. Microw. Symp. (IMS'17), Honolulu, Hawaii, June 2017

21. J. Naqui, M. Durán-Sindreu, F. Martín, Alignment and position sensors based on split ring resonators, Sensors 12, $11790(2012)$

22. A. Karami-Horestani, C. Fumeaux, S.F. Al-Sarawi, D. Abbott, Displacement sensor based on diamond-shaped tapered split ring resonator, IEEE Sens. J. 13, 1153 (2013)

23. A.K. Horestani, J. Naqui, D. Abbott, C. Fumeaux, F. Martín, Two-dimensional displacement and alignment sensor based on reflection coefficients of open microstrip lines loaded with split ring resonators, Electron. Lett. 50, 620 (2014)

24. J. Naqui, F. Martín, Transmission lines loaded with bisymmetric resonators and their application to angular displacement and velocity sensors, IEEE Trans. Microw. Theor. Technol. 61, 4700 (2013)

25. J. Naqui, F. Martín, Angular displacement and velocity sensors based on electric-LC (ELC) loaded microstrip lines, IEEE Sens. J. 14, 939 (2014)

26. J. Naqui, J. Coromina, A. Karami-Horestani, C. Fumeaux, F. Martín, Angular displacement and velocity sensors based on coplanar waveguides (CPWs) loaded with S-shaped split ring resonator (S-SRR), Sensors 15, 9628 (2015)

27. L. Su, J. Mata-Contreras, J. Naqui, F. Martín, Splitter/ combiner microstrip sections loaded with pairs of complementary split ring resonators (CSRRs): modeling and optimization for differential sensing applications, IEEE Trans. Microw. Theor. Technnol. 64, 4362 (2016)

28. L. Su, J. Mata-Contreras, J. Naqui, F. Martín, Configurations of splitter/combiner microstrip sections loaded with stepped impedance resonators (SIRs) for sensing applications, Sensors 16, 2195 (2016), doi:10.3390/s16122195

29. J. Naqui, F. Martín, Application of broadside-coupled split ring resonator (BC-SRR) loaded transmission lines to the design of rotary encoders for space applications, IEEE MTTS Int. Microw. Symp. (IMS'16), San Francisco, 2016

30. J. Mata-Contreras, C. Herrojo, F. Martín, Application of split ring resonator (SRR) loaded transmission lines to the design of angular displacement and velocity sensors for space applications, IEEE Trans. Microw. Theory Techn. (to be published)

31. S. Preradovic, N.C. Karmakar, Chipless RFID: bar code of the future, IEEE Microw. Mag. 11, 87 (2010)

32. S. Preradovic, N.C. Karmakar, Multiresonator-based chipless RFID: barcode of the future (Springer, 2011)

33. S. Preradovic, I. Balbin, N.C. Karmakar, G.F. Swiegers, Multiresonator-based chipless RFID system for low-cost item tracking, IEEE Trans. Microw. Theor. Technol. 57, 1411 (2009) 
34. S. Preradovic, N.C. Karmakar, Design of chipless RFID tag for operation on flexible laminates, IEEE Anten. Wirel. Propag. Lett. 9, 207 (2010)

35. C. Herrojo, J. Naqui, F. Paredes, F. Martín, Spectral signature barcodes based on s-shaped split ring resonators (SSRR), EPJ Appl. Metamater. 3, 1 (2016)

36. C. Herrojo, J. Naqui, F. Paredes, F. Martín, Spectral signature barcodes implemented by multi-state multiresonator circuits for chipless RFID tags, in: IEEE MTT-S International Microwave Symposium (IMS'16), San Francisco, 2016

37. C. Herrojo, F. Paredes, J. Mata-Contreras, S. Zuffanelli, F. Martín, Multi-state multi-resonator spectral signature barcodes implemented by means of S-shaped Split Ring Resonators (S-SRR), IEEE Trans. Microw. Theor. Technol. 65, 2341 (2017)

38. O. Rance, R. Siragusa, P. Lemaître-Auger, E. Perret, Toward RCS magnitude level coding for chipless RFID, IEEE Trans. Microw. Theor. Technol. 64, 2315 (2016)

39. J. McVay, A. Hoorfar, N. Engheta, Space-filling curve RFID tags, in: Proceedings of 2006 IEEE Radio Wireless Symposium, 2006, pp. 199-202

40. I. Jalaly, D. Robertson, Capacitively-tuned split microstrip resonators for RFID barcodes, in: Proceedings of European Microwave Conference, 2005, pp. 4-7

41. H.-S. Jang, W.-G. Lim, K.-S. Oh, S.-M. Moon, J.-W. Yu, Design of low-cost chipless system using printable chipless tag with electromagnetic code, IEEE Microw. Wireless Compon. Lett. 20, 640 (2010)

42. A. Vena, E. Perret, S. Tedjini, A fully printable chipless RFID tag with detuning correction technique, IEEE Microw. Wirel. Compon. Lett. 22, 209 (2012)

43. A. Vena, E. Perret, S. Tedjini, Design of compact and autocompensated single-layer chipless RFID tag, IEEE Trans. Microw. Theor. Technol. 60, 2913 (2012)

44. A. Vena, E. Perret, S. Tedjini, High-capacity chipless RFID tag insensitive to the polarization, IEEE Trans. Ant. Propag. 60, 4509 (2012)

45. M.A. Islam, N.C. Karmakar, A novel compact printable dualpolarized chipless RFID system, IEEE Trans. Microw. Theor. Technol. 60, 2142 (2012)

46. A. Vena, E. Perret, S. Tedjini, Chipless RFID tag using hybrid coding technique, IEEE Trans. Microw. Theor. Technol. 59, 3356 (2011)
47. A. Vena, E. Perret, S. Tedjini, A compact chipless RFID tag using polarization diversity for encoding and sensing, in: 2012 IEEE Int. Conf. RFID, 2012, pp. 191-197

48. I. Balbin, N.C. Karmakar, Phase-encoded chipless RFID transponder for large scale low cost applications, IEEE Microw. Wirel. Comp. Lett. 19, 509 (2009)

49. S. Genovesi, F. Costa, A. Monorchio, G. Manara, Chipless RFID tag exploiting multifrequency delta-phase quantization encoding, IEEE Ant. Wirel. Propag. Lett. 15, 738 (2015)

50. C. Herrojo, J. Mata-Contreras, F. Paredes, F. Martín, NearField chipless RFID encoders with sequential bit reading and high data capacity, in: IEEE MTT-S Int. Microw. Symp. (IMS'17), Honolulu, Hawaii, 2017

51. C. Herrojo, J. Mata-Contreras, F. Paredes, F. Martín, Chipless RFID tags based on metamaterial concepts, in: The 11th International Congress on Engineered Material Platforms for Novel Wave Phenomena (Metamaterials), 2017

52. H. Chen, L. Ran, J. Huangfu, X. Zhang, K. Chen, T.M. Grzegorczyk, J.A. Kong, Left-handed materials composed of only S-shaped resonators, Phys. Rev. E. 70, 1 (2004)

53. H. Chen, L. Ran, J. Huangfu, X. Zhang, K. Chen, T.M. Grzegorczyk, J.A. Kong, Negative refraction of a combined double S-shaped metamaterial, Appl. Phys. Lett. 86, 151909 (2005)

54. H. Chen, L.-X. Ran, H.-F. Jiang Tao, X.-M. Zhang, K.-S. Cheng, T.M. Grzegorczyk, J.A. Kong, Magnetic properties of S-shaped split ring resonators, Prog. Electromagn. Res. 51, 231 (2005)

55. E. Shamonina, V.A. Kalinin, K.H. Ringhofer, L. Solymar, Magneto-inductive waveguide, Electron. Lett. 38, 371 (2002)

56. E. Shamonina, V.A. Kalinin, K.H. Ringhofer, L. Solymar, Magneto-inductive waves in one, two and three dimensions, J. Appl. Phys. 92, 6252 (2002)

57. M.C.K. Wiltshire, E. Shamonina, I.R. Young, L. Solymar, Dispersion characteristics of magneto-inductive waves: comparison between theory and experiment, Electron. Lett. 39, 215 (2003)

58. E. Shamonina, L. Solymar, Properties of magnetically coupled metamaterial elements, J. Magn. Magn. Mat. 300, 38 (2006)

59. R.R.A. Syms, E. Shamonina, V. Kalinin, L. Solymar, A theory of metamaterials based on periodically loaded transmission lines: interaction between magnetoinductive and electromagnetic waves, J. Appl. Phys. 97, 064909 (2005)

60. M.J. Freire, R. Marqués, F. Medina, M.A.G. Laso, F. Martin, Planar magnetoinductive wave transducers: theory and applications, Appl. Phys. Lett. 85, 4439 (2004)

Cite this article as: Cristian Herrojo, Javier Mata-Contreras, Ferran Paredes, Ferran Martín, High data density and capacity in chipless radiofrequency identification (chipless-RFID) tags based on double-chains of S-shaped split ring resonators (S-SRRs), EPJ Appl. Metamat. 2017, 4,8 